\title{
Centennial wisdom and modern science: A challenging and promising combination
}

\author{
Carolina Lessa-Aquino
}

Aquino CL. Centennial wisdom and modern science: A challenging and promising combination. Curr Res Integr Med 2017;2(3):30.

$\mathrm{T}$ aditional medicine has achieved remarkable popularity over the last decades. Experience-based knowledge has passed down through generations and the practical wisdom, whether explicable or not, has been successfully used in the maintenance of physical and mental health for hundreds of years. Originally inherent to different cultures, it is now continuously advancing worldwide. Along with this progress, the scientific community puts increasing efforts to help building a strong and a highquality science in the field.

In 1946, the Constitution of the World Health Organization defined health as "a state of complete physical, mental and social well-being and not merely the absence of disease or infirmity". In countries where Western conventional medicine prevails, the inclusion of the so-called alternative or complementary medicine practices in the national health care system is a turning point. Although challenging, there is considerable evidence that it may reduce medical expenditure while also playing a key role in emphasizing the holistic health perspective stated by WHO.

In Latin America, Brazil and Cuba are in the vanguard of this movement. In both countries, national programs for integrating traditional medicine into the formal health system were implemented in the end of XX century. Currently, Cuba has a significant production of natural and homeopathic medication. Brazil, on the other hand, is the regional leader in integrative medicine initiatives. Fourteen traditional practices have been integrated into Brazilian Unified Health System (SUS), including traditional Chinese medicine - acupuncture, homeopathy, anthroposophical medicine, hydrotherapy/crenotherapy, phytotherapy, medicinal plants, ayurveda, chyropraxy, yoga and meditation. The incorporation of integrative practices into basic health systems not only valorizes the country's cultural roots but also favors cultural exchange. Other Latin countries, such as Argentina, Chile, Mexico, Colombia, Bolivia and Venezuela are also moving towards integration, but are presently at earlier stages.

The movement for integrative medicine is global. Together with it comes the necessity to fit this centennial wisdom into the contemporary scientific methodology that rules modern science. In order to be labeled as safe, effective and trusted, traditional practices should be submitted to extensive scientific examination, which is far from trivial when it comes to a holistic perspective. Nevertheless, scientists all over the world are in a joint effort to provide the community with standard high-quality research on integrative medicine. A quick search on PubMed with the query terms "traditional medicine" or "alternative medicine" or "complementary medicine" generates, in August 2017, approximately 30,000 results, half of which $(\sim 17,000)$ were published in the last decade and one-third $(\sim 10,000)$ in the last 5 years.

I invite more of our esteemed colleagues to contribute to efforts to strengthen this global trend. Current Research Integrative Medicine encourages the submission of original research articles, clinical studies, case reports and review articles describing a wide range of topics related to human health. Whether focusing on traditional or conventional medicine, each and every study regarding aspects that integrate to affect a person's health is welcome.

Instituto Oswaldo Cruz - Fiocruz

Correspondence: Dr. Carolina Lessa-Aquino, Instituto Oswaldo Cruz/IOC/FIOCRUZ, Manguinhos - Rio de Janeiro - RJ - Brazil, e-mail claquino2011@gmail.com Received: August 21, 2017, Accepted: August 22, 2017, Published: August 31, 2017 